\title{
Blue diode laser versus traditional infrared diode laser and quantic molecular resonance scalpel: clinical and histological findings after excisional biopsy of benign oral lesions
}

Margherita Gobbo

Rossana Bussani

Giuseppe Perinetti

Katia Rupel

Lorenzo Bevilacqua

Giulia Ottaviani

Matteo Biasotto 


\title{
Blue diode laser versus traditional infrared diode laser and quantic molecular resonance scalpel: clinical and histological findings after excisional biopsy of benign oral lesions
}

\author{
Margherita Gobbo, ${ }^{\mathrm{a}, \mathrm{b}}$ Rossana Bussani, ${ }^{\mathrm{b}, \mathrm{c}}$ Giuseppe Perinetti, ${ }^{\mathrm{b}}$ Katia Rupel, ${ }^{\mathrm{a}, \mathrm{b}}$ Lorenzo Bevilacqua, \\ Giulia Ottaviani, ${ }^{a, b, *}$ and Matteo Biasotto ${ }^{a, b}$ \\ ${ }^{a}$ Division of Oral Medicine and Pathology, Dental Clinic, Ospedale Maggiore, Trieste, Italy \\ bUniversity of Trieste, Department of Surgical, Medical and Health Sciences, Trieste, Italy \\ Institute of Pathologic Anatomy, Ospedale di Cattinara, Trieste, Italy
}

\begin{abstract}
This study aims to compare the use of the innovative blue diode laser (BLUE group) with two traditional surgical techniques: the infrared diode laser (IR group) and the quantic molecular resonance scalpel (QMR group) in the excision of benign oral lesions. Ninety-three patients underwent surgical excision of a benign oral lesion and were followed up for 30 days for pain ( 0 to 10 visual analogue scale), bleeding, and painkillers' assumption (yes/no). A blind pathologist evaluated the thermal damage along the cutting margin. Although referred pain was lowest in the BLUE group from day 7 on $(p<0.05)$, all patients referred minimum discomfort after surgery. The BLUE group reported minimum bleeding and necessity of sutures $(p<0.000)$. The QMR group showed the highest bleeding during surgery $(p<0.000)$, while after 14 and 30 days no patient bled. Most of the patients in all groups did not need painkillers. The lowest thermal damage $(p<0.000)$ was found in the BLUE group $(71.3 \pm 51.8 \mu \mathrm{m})$, whereas the IR group proved the highest $(186.8 \pm 82.7 \mu \mathrm{m}) \mathrm{com}$ pared both with the BLUE and QMR $(111.4 \pm 55.4 \mu \mathrm{m})$ groups. All the techniques allowed correct histological sampling. All the experimented techniques offer interesting advantages, although the blue laser minimizes risk of bleeding with limited thermal damage. ( $) 2017$ Society of Photo-Optical Instrumentation Engineers (SPIE) [DOI: 10.1117/1.JBO.22.12 .121602]
\end{abstract}

Keywords: blue laser; oral surgery; biopsy; bleeding; healing; thermal damage.

Paper 170099SSPR received Mar. 3, 2017; accepted for publication May 22, 2017; published online Jul. 12, 2017; corrected Feb. 20, 2019.

\section{Introduction}

In recent years, the use of surgical instruments based on new technologies has largely influenced the medical practice. In the oral medicine and pathology fields, these techniques have improved the management of various affections, including the excisional biopsy of benign lesions. ${ }^{1}$

Specialists often deal with local complications such as hemorrhage during surgery or high referred pain during the postoperative period. Traditionally, interventions are performed with the cold blade, a highly precise, quick, and cheap device; unfortunately, it is sometimes connected to difficulties in bleeding control—with consequent scarce field visibility—and often requires the execution of sutures. Moreover, the use of a scalpel is usually operator-dependent since risky anatomical structures may be encountered and damaged. ${ }^{2}$

Studies regarding the use of electroscalpels, quantic molecular resonance (QMR) scalpels, and laser scalpels have provided a growing literature on the topic. ${ }^{3}$

The QMR scalpel is an electrocautery technique that applies high-frequency waves. The cut is achieved by the explosion of infracellular and intracellular liquids that resonate with a special frequency. A temperature lower than $45 \mathrm{deg}$ results in

*Address all correspondence to: Giulia Ottaviani, E-mail: giulia.ottaviani@ phd units.it nontraumatic cutting, gentle coagulation, and an absence of thermal injury. Denaturation of fibrinogen at $63^{\circ} \mathrm{C}$ generates coagulation, and the result is the elimination of scar tissue or keloid, with considerable aesthetic benefits and a reduction in postoperative swelling and pain. ${ }^{4}$

Several advantages are related to the use of laser scalpels and to the possibility of combining various wavelengths and properties. For example, lasers allow reduction of the operating time, the possibility to combine the cut with the coagulation of the target tissue, a reduced use of sutures and pain, the disinfection of the field, and a better and faster healing process, so avoiding the utilization of drugs, giving patients' greater comfort during the postoperating time. ${ }^{5}$ Many procedures may benefit from the introduction of these devices, including incisional and excisional biopsies, aesthetic surgery, and functional surgery of oral soft tissues (i.e., frenulectomy). ${ }^{6}$ Both the QMR and the lasers allow minimally invasive surgery, based on the reduction of operatory trauma, preservation of anatomical structures, and limited pain. These devices have several advantages, as compared with traditional techniques, such as the reduced need for anesthesia, which in some cases can be completely avoided, and the limited necessity of stitches after intervention. Moreover, analyzing the necessity of painkillers' assumption after surgery, the QMR and lasers devices are related to a reduced need for

$1083-3668 / 2017 / \$ 25.00$ (c) 2017 SPIE 
postoperative pharmacological therapy. ${ }^{7}$ For all the above-mentioned reasons, QMR and lasers may be considered useful in dentistry, including the treatment of pediatrics. ${ }^{8,9}$

However, most of the published clinical works on oral surgery only consider the surgical performances of lasers emitting in the infrared (IR) wavelength spectrum, ${ }^{10}$ while recently a blue laser has been introduced in the surgical field. ${ }^{11,12}$ The blue laser does not work through water absorption but rather on the absorption of melanin and hemoglobin chromophores. This specific feature allows obtaining specific advantages in the surgical field: absence of bleeding during surgery, with optimum visibility of the operating field, and less necessity to execute sutures. Since bleeding is usually absent and visibility is optimal, this laser device is associated with shortened procedures and absent or limited pain, as well as functional and aesthetic high success rates. The absence of bleeding, the optimal visibility, and the fact that the patient feels at ease make the operator feel comfortable during surgery. Other characteristics are: a higher energy coefficient (compared with the IR laser) and increased antiseptic and photobiomodulating properties. ${ }^{13,14}$

Since the beginning of the "biomedical optics era" in oral surgery, one of the major concerns of surgeons was the risk of damaging tissue samples, thus hindering the analysis of the pathologist. Nevertheless, recent techniques allow excellent preservation of the sample, minimal thermal damage along the cutting margin, slight modification of molecular structures, and few or no modifications at the cellular level. This implies that the pathologist can easily analyze samples and that these techniques can be considered suitable both for benign and malignant conditions, where surgical margins are of fundamental importance. ${ }^{15}$

\section{Aim of the Study}

The main objective of this study is the comparison of the new blue laser device with two electrosurgical techniques, QMR and IR laser devices, already employed in clinical practice, for the excision of benign oral lesions in different oral sites. Pain, bleeding, and the necessity of painkillers were monitored over time, and a blind pathologist quantified the thermal damage induced by surgery.

\section{Materials and Methods}

This study was conducted at the "Oral Medicine and Pathology Unit" of the Dental Clinic (Ospedale Maggiore, Trieste, Italy), after approval by the ethical committee (Comitato etico di ateneo, n.1247/2014 prot. 25138. Verbale n. 73, adunanza 12.09.2016).

A total of 93 patients (51 females and 34 males) were enrolled in the present prospective study; group BLUE was composed of 39 subjects, group IR of 27 subjects, and group QMR of 27 subjects. The mean age was $59.1 \pm 16.5$ years. Sex and gender were equally distributed among groups.

Patients were enrolled according to the following criteria:

- isolated and well demarcated oral lesion;

- easy surgical access and good visibility;

- absence of scars due to previous interventions;

- no clinical history of difficulty in wound healing;

- no assumption of drugs that influence the blood flow or bleeding diatheses.

A total of 93 patients were randomly (one each consecutively) assigned to the following groups:
- Group 1 (BLUE group) was composed of 39 subjects, treated by InGaN diode laser (class IV, K-Laser Blue, Eltech S.r.l., Treviso, Italy) with the following parameters: 445-nm wavelength, 2-W peak power, $t$-on $20 \mathrm{~ms}, t$-off $8 \mathrm{~ms}, 1.4-\mathrm{W}$ average power, and $320-\mu \mathrm{m}$ fiber.

- Group 2 (IR group) was composed of 27 subjects, treated by a diode laser (class IV, K-Laser K-Series, Eltech S.r.l., Treviso, Italy) with the following parameters: 970-nm wavelength, 6.0-W peak power, pulsed modality (33\% duty cycle), 10-Hz frequency, 2-W average power, and $320-\mu \mathrm{m}$ fiber.

- Group 3 (QMR) was composed of 27 subjects treated by an electroscalpel, specifically a QMR scalpel, produced by VESALIUS (Telea Electronic Engineering S.r.l.) used in "cut and coagulate" modality with a thin, straight electrode.

All patients were visited at the Oral Medicine and Pathology Unit by a third-year oral surgery major. When an apparently benign oral lesion was evidenced, the same operator performed an excisional biopsy. Before performing the biopsy and during each follow-up, a photo of the lesion's area was taken and its dimension was measured using a graduated probe. Patients were excluded from the study before biopsy in cases of painful lesion, bleeding lesion, assumption of painkillers on the day of the biopsy, and histological report referring to dysplastic or neoplastic condition.

All biopsies were performed after obtaining written informed consent of the patients and using local anesthesia with mepivacaine and adrenaline $(1: 100.000)$. After biopsy, stitches were put in only in cases of bleeding. Samples were sent to a dedicated oral pathologist for histopathological examination (hematoxylin and eosin staining) with the request of quantifying the maximum thermal damage of the specimen (expressed in microns) along the cutting margin. The pathologist who examined the specimen was blinded in regard to the employed technique.

All patients were followed up for pain using a 0-to-10 visual analogue scale (VAS), for bleeding (yes/no), and for painkillers' assumption (yes/no) on the first day (T0) and 2 (T2), 7 (T7), 14 (T14), and 30 (T30) days after biopsy.

Table 1 Frequencies (as number of cases) of the different sites among the groups.

\begin{tabular}{lccr} 
& \multicolumn{3}{c}{ Group } \\
\cline { 2 - 4 } Site & BLUE & IR & QMR \\
\hline Lip & 14 & 14 & 6 \\
Cheek & 13 & 6 & 11 \\
Palate & 1 & 2 & 1 \\
Tongue & 9 & 3 & 7 \\
Floor of the mouth & 1 & 0 & 1 \\
Gingiva & 1 & 2 & 1 \\
\hline Diff. & & 0.522 & \\
\hline
\end{tabular}


Table 2 Frequencies (as number of cases) of the different diagnoses among the groups.

\begin{tabular}{lccc}
\hline & \multicolumn{3}{c}{ Group } \\
\cline { 2 - 4 } Category & BLUE & IR & QMR \\
\hline Diagnosis & 22 & 13 & 20 \\
Fibroma & 5 & 5 & 0 \\
Angiofibroma & 1 & 0 & 1 \\
Epulis & 5 & 3 & 3 \\
Mucocele & 2 & 3 & 2 \\
Papilloma & 3 & 2 & 0 \\
Angioma & 1 & 0 & 0 \\
Amalgam tattoo & 0 & 1 & 0 \\
Papillae & 0 & 0 & 1 \\
Lipoma & & 0.436 & \\
\hline Diff. & & & \\
\hline
\end{tabular}

\section{Statistical Analysis}

The SPSS software, version 13.0 (SPSS ${ }^{\circledR}$ Inc., Chicago, Illinois) was used to perform the statistical analyses.

The significance of the differences among the groups in the frequencies of the categories within diagnosis and site were evaluated by means of a Chi-squared test.

After testing the normality of the data with the Shapiro-Wilk test and Q-Q normality plots and the equality of variance among the datasets using a Levene test, nonparametric methods were used for data analysis.

A Kruskal-Wallis test was used to assess the significance of the differences of VAS among the groups at each of the time points. The significance of the differences in the perceived pain after biopsy (VAS) over time within the groups was evaluated using a Friedman test.

The Chi-square test was used to assess the significance of the differences of bleeding and painkiller assumption among the groups at each of the time points. The significance of the differences in bleeding and painkiller assumption over time within the groups was evaluated using a Cochrane test.

The Kruskal-Wallis test was used to assess the significance of the differences in the dimension of the thermal damage among the groups.

Whenever necessary, pairwise comparisons were performed by a Bonferroni corrected Mann Whitney U-test.

A $p$ value of less than 0.05 was used for the rejection of the null hypothesis.

Table 3 The VAS (0 to 10 score), bleeding (0, no; 1 , yes), and painkiller assumption ( 0 , no; 1 , yes) in the different groups over time.

\begin{tabular}{|c|c|c|c|c|c|c|c|}
\hline \multirow[b]{2}{*}{ Parameter } & \multirow[b]{2}{*}{ Group } & \multicolumn{5}{|c|}{ Time } & \multirow[b]{2}{*}{ Diff. } \\
\hline & & TO & $\mathrm{T} 2$ & T7 & $\mathrm{T} 14$ & Т30 & \\
\hline \multirow[t]{4}{*}{ VAS } & BLUE & $0.0 \pm 0.0$ & $0.7 \pm 1.7$ & $0.2 \pm 0.5$ & $0.0 \pm 0.0$ & $0.1 \pm 0.5$ & $<0.05$ \\
\hline & IR & $0.4 \pm 1.5$ & $0.3 \pm 0.6$ & $0.3 \pm 0.7$ & $0.3 \pm 0.8$ & $0.0 \pm 0.0$ & NS \\
\hline & QMR & $0.0 \pm 0.0$ & $0.7 \pm 1.3$ & $0.7 \pm 1.2$ & $0.2 \pm 0.5$ & $0.0 \pm 0.0$ & $<0.05$ \\
\hline & Diff. & NS & NS & $<0.05$ & $<0.05$ & NS & \\
\hline \multirow[t]{4}{*}{ Bleeding } & BLUE & 2 & 3 & 1 & 0 & 0 & NS \\
\hline & IR & 5 & 1 & 1 & 0 & 0 & $<0.05$ \\
\hline & QMR & $21^{a, b}$ & 2 & 1 & 0 & 0 & $<0.05$ \\
\hline & Diff. & $<0.05$ & NS & NS & - & - & \\
\hline \multirow[t]{4}{*}{ Painkiller assumption } & BLUE & 1 & 2 & 0 & 0 & 0 & NS \\
\hline & IR & 0 & 5 & 3 & 0 & 0 & $<0.05$ \\
\hline & QMR & 0 & 6 & 0 & 0 & 0 & $<0.05$ \\
\hline & Diff. & NS & NS & $<0.05$ & - & - & \\
\hline
\end{tabular}

Note: Diff., significance of the difference among the groups at each time point or over time within each group. VAS presented as mean \pm SD. Bleeding and painkiller assumption presented as number of positive cases.

BLUE treatment, $n=39$, IR treatment, $n=27$, QNR treatment, $n=27$.

aSignificantly different than BLUE treatment.

bSignificantly different than IR treatment. 


\section{Results}

A total of 93 benign oral lesions were diagnosed following histopathological analysis, including fibromas, angiofibromas, epulis, mucoceles, papillomas, angiomas, amalgam tattoos, papillae, and lipomas. Lesions affected various sites in the oral cavity including lips, cheeks, palate, tongue, floor of the mouth, and gingiva. Frequencies (as number of cases) of the different diagnoses or sites among the groups are reported in Tables 1 and 2. Lesions were equally distributed among groups according to the Chi-square test.

Table 4 The thermal damage score $(\mu \mathrm{m})$ among the different groups.

\begin{tabular}{lccc}
\hline & \multicolumn{3}{c}{ Thermal damage $(\mu \mathrm{m})$} \\
\cline { 2 - 4 } Group & Mean \pm SD & Median & Min-max \\
\hline BLUE $(n=24)$ & $71.3 \pm 51.8$ & 54.4 & $27.2-268.7$ \\
IR $(n=24)$ & $186.8 \pm 82.7^{\mathrm{a}}$ & 173.3 & $50.0-406.9$ \\
QMR $(n=25)$ & $111.4 \pm 55.4^{\mathrm{a}, \mathrm{b}}$ & 95.9 & $32.5-256.4$ \\
\hline Diff. & & $p<0.05$ \\
\hline
\end{tabular}

Note: Min-max, minimum maximum values; Diff., significance of the difference among the groups.

aSignificantly different than IR treatment.

bSignificantly different than BLUE treatment.
The variation of VAS, bleeding, and painkiller assumption in the different groups over time is reported in Table 3.

After surgery, in all groups, minimum pain was reported during follow-ups with no differences among groups after 2 days, but a lower reported VAS for the BLUE group at T7 and T14. All patients were completely asymptomatic at T30.

Regarding bleeding, the patients in the QMR group bled during surgery significantly more than those in the BLUE and IR groups; the number of cases treated with sutures after interventions were 5, 4, and 20 in the BLUE, IR, and QMR groups, respectively $(p<0.05)$.

No differences were evidenced regarding postoperative bleeding comparing the three techniques. At T14 and T30, no patients referred bleeding in any group.

The necessity of painkiller assumption was higher in groups IR and QMR at T2, although not statistically significantly. At T7, only three patients in the IR group had assumed painkillers. At T14 and T30, no patients had taken painkillers.

Mean dimension of the lesions was $5.7 \pm 2.4 \mu \mathrm{m}$ in the BLUE group, $6.0 \pm 2.2 \mu \mathrm{m}$ in the IR group, and $6.9 \pm 2.9 \mu \mathrm{m}$ in the QMR group. Lesions were equally distributed among groups according to dimension (ANOVA test: NS).

The thermal damage $(\mu \mathrm{m})$ induced by each device is reported in Table 4.

A lower thermal damage was found in the BLUE group (Fig. 1), whereas the IR group (Fig. 2) proved worse than both BLUE and QMR (Fig. 3).
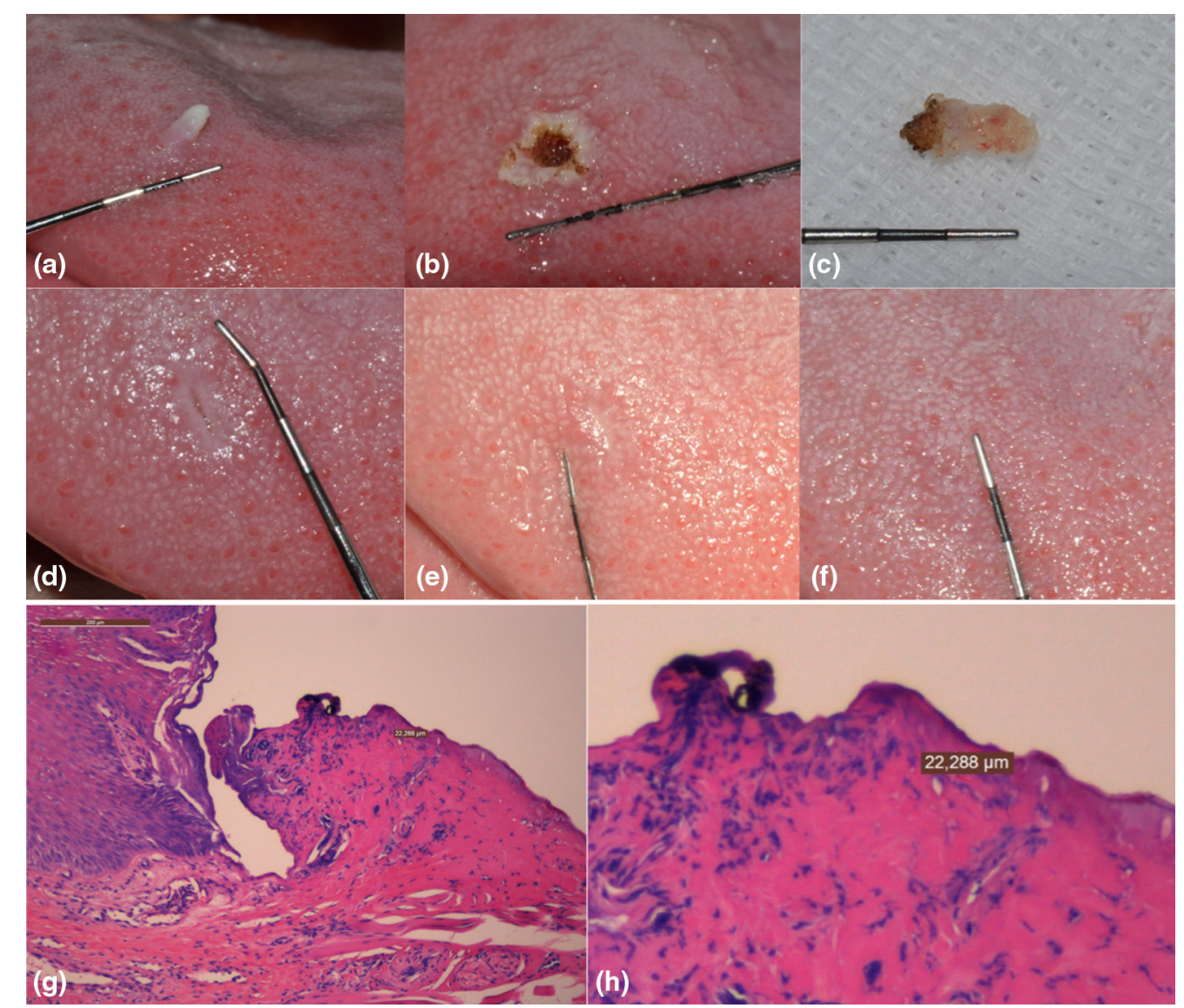

Fig. 1 Intervention performed with BLUE laser: excision of papilloma of tongue dorsum. (a) Preoperative,

(b) excision at T0, (c) tissue sample at T0, (d) T7 follow-up, (e) T14 follow-up, (f) T30 follow-up, and (g) histological evaluation of the lesion and (h) magnification of thermal damage measurement $(\mu \mathrm{m})$. 


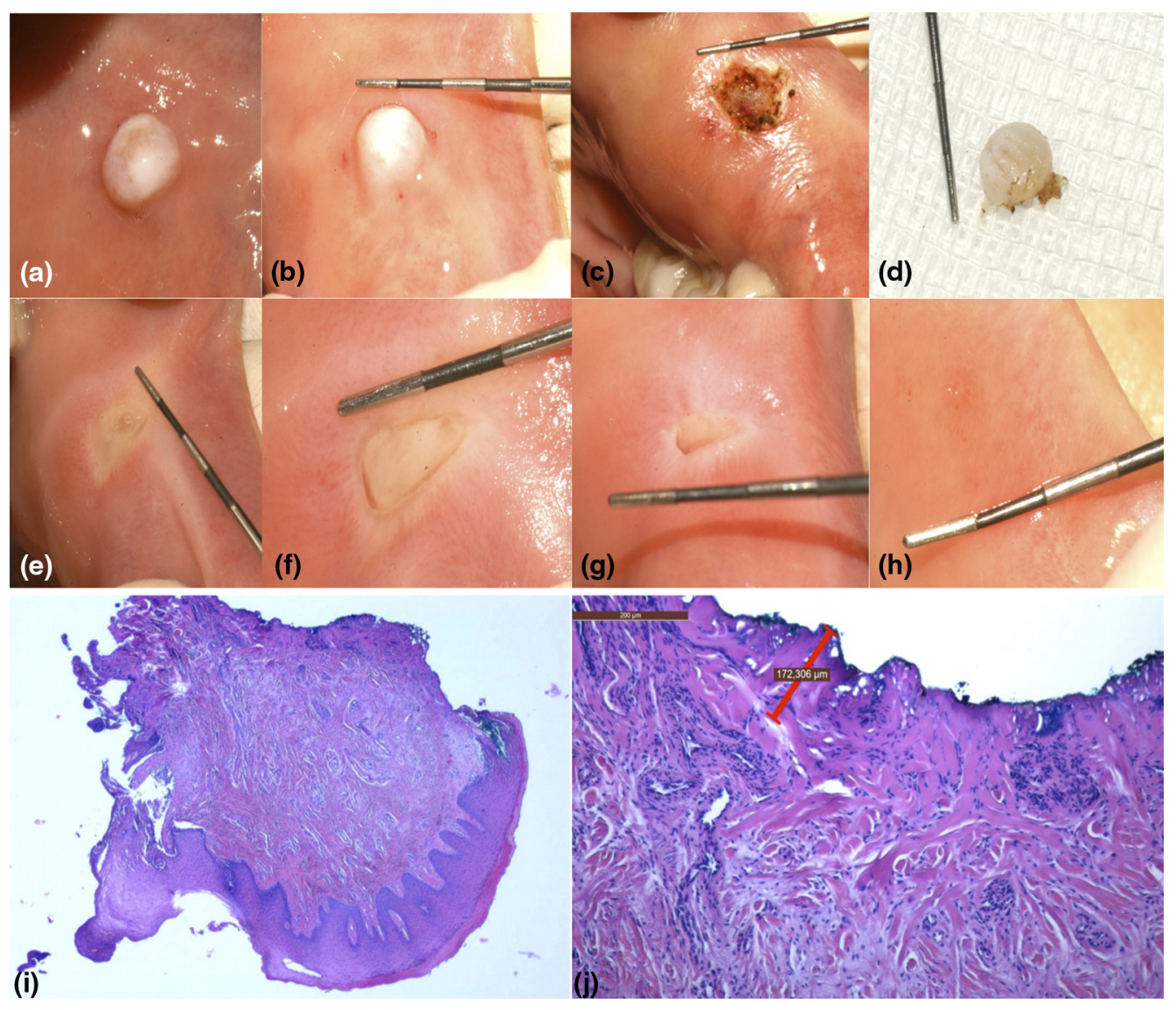

Fig. 2 Intervention performed with IR laser: excision of fibroma on left cheek with QMR. (a) Preoperative, (b) lesion's dimension, (c) excision at T0, (d) tissue sample at T0, (e) T2 follow-up, (f) T7 follow-up, (g) T14 follow-up, (h) T30 follow-up, (i) histological evaluation of the lesion, and (j) magnification and thermal damage measurement $(\mu \mathrm{m})$.

In 20 samples, the pathologist did not calculate the thermal damage either because it was not evaluable or because it was not recognizable in the sample.

Specifically, it was not evaluable in smaller lesions (mean dimension $4.6 \pm 2.1 \mu \mathrm{m})$ and not recognizable with bigger ones (mean dimension $5.5 \pm 2.1 \mu \mathrm{m}$ ). In all cases, the pathologist had no difficulties in performing a histological diagnosis following the use of an electrosurgical device.

\section{Discussion}

The results of this study underline that, despite the surgical performance differences, both laser devices and QMR can be successfully employed in the management of soft tissue benign oral lesions during daily clinical practice.

We have considered a variety of benign lesions, which are usually managed with traditional scalpels. Many studies have compared the use of the traditional cold blade with more "technological" devices, such as lasers and the QMR, proving that, although quick, the cold blade is burdened by a series of disadvantages. These include bleeding, scarce visibility, and difficult excision in some narrow anatomical areas, such as the soft palate and posterior tongue. In addition, when used by nonexpert operators, the cold blade can be hazardous, causing unexpected damage to anatomical structures such as nerves or blood vessels. ${ }^{16}$ Moreover, a switch or a pedal usually activates lasers and QMR. This option is comfortable for operators, who can autonomously activate and deactivate the device for any kind of reason, including the necessity of changing the cutting position or the incident angle. In fact, an orthogonal cut is usually more effective, above all, for the lasers. This implies that the tip must be tilted following the shape and position of the lesion. In addition, in cases of sudden and unexpected movement of the patient, the device can be quickly switched off by releasing the activating switch or pedal, avoiding any damage to anatomical structures. Using a cold blade, the position of the blade is not always comfortable and the handle is less ergonomic. Moreover, in cases of unexpected movement of the patient, the risk of hurting neighboring areas is quite high. ${ }^{17}$

However, with this study, we decided to test a new device, the blue diode laser, since it could represent an interesting and challenging device for clinicians, especially working in the oral surgery field, due to its peculiar characteristics. For this reason, we analyzed its clinical characteristics and performance parameters, comparing them with the highest "technological" devices (a diode IR laser and QMR scalpel), nowadays routinely employed to biopsy benign oral lesions belonging to different anatomical sites.

The complete absence of bleeding in the BLUE group can be considered its most important advantage, and, according to our results, it reached the highest rate compared with IR and QMR groups. ${ }^{18}$ To note, more than $77 \%$ of lesions treated by QMR bled during surgery. Consequently, the blue laser device could be considered ideal tool for the treatment of highly vascularized lesions, such as angiomas, artero-venous malformations, 

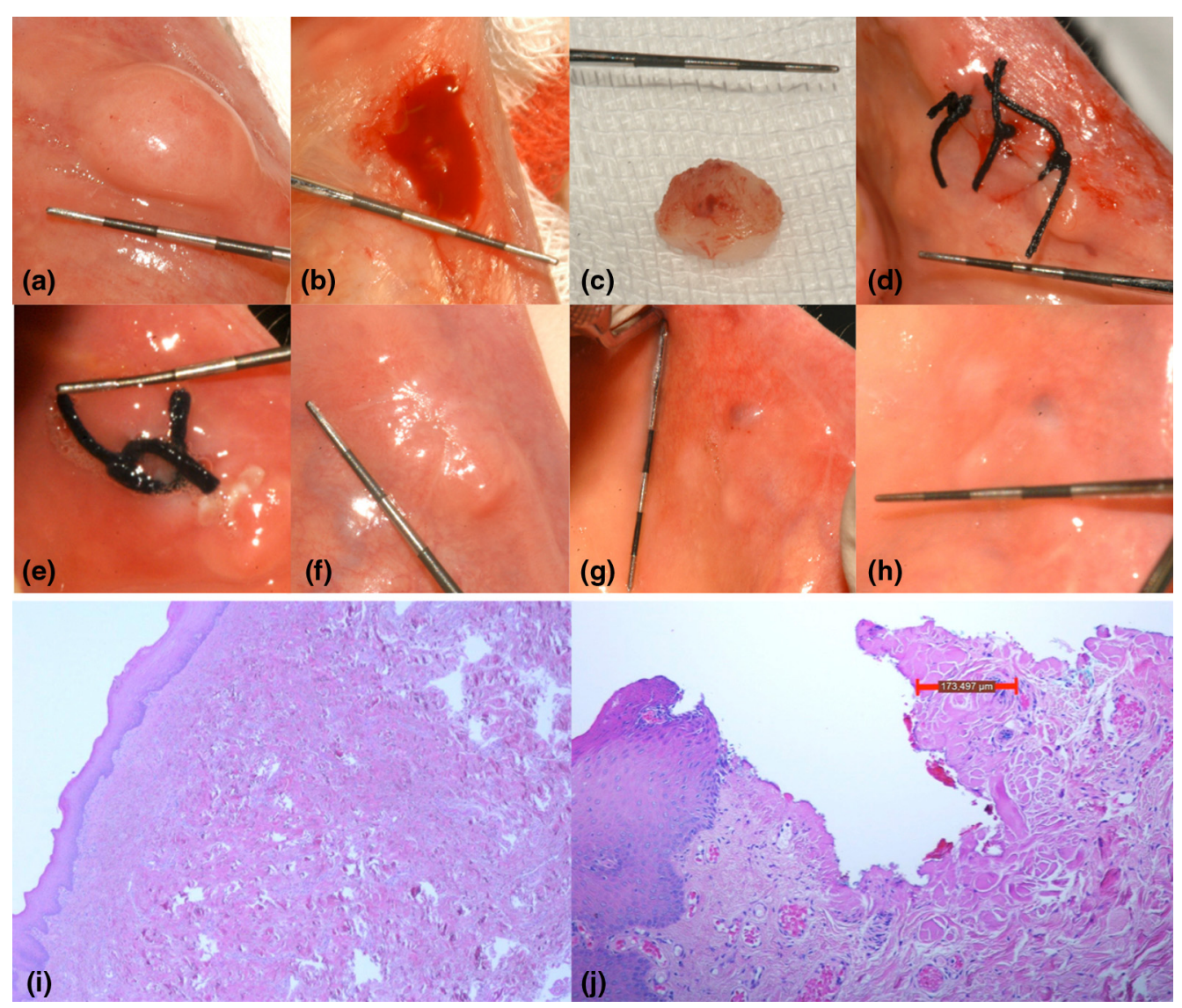

Fig. 3 Intervention performed with QMR scalpel: excision of an angiofibroma from lower lip. (a) Preoperative, (b) excision at T0, (c) tissue sample at T0, (d) end of surgery at T0, (e) T2 followup, (f) T7 follow-up, (g) T14 follow-up, (h) T30 follow-up, (i) histological evaluation of the lesion, and (j) magnification and thermal damage measurement $(\mu \mathrm{m})$.

pyogenic granulomas, and varicosities. In addition, together with IR lasers, it should also be preferred to QMR in areas where large vessels can be encountered, such as lips, floor of the mouth, and retrocommissural area. Another clinical application could be represented, with a higher performance rate compared with the IR laser, by the photocoagulation of vascular lesions, when they represent for patients an aesthetic concern rather than a pathological condition, without necessity of anesthesia and with optimal aesthetic results. With QMR devices, it would not be possible to insert a dedicated tip into the lesion and to perform its photocoagulation. ${ }^{19,20}$

In this study, the performing of stitches after excisional biopsy was restricted to lesions that bled during intervention. Once again, in the BLUE group, stitches were applied to only $5 \%$ of patients and to $18 \%$ in the IR group, whereas the vast majority of patients treated with QMR (74\%) needed sutures. ${ }^{21}$ Secondary intention healing favors the achievement of a satisfactory tissue healing after biopsy. First, it reduces the duration of intervention because putting stitches can sometimes be hard in certain anatomical areas, such as the oropharyngeal and palatal zones, as well as on the gingiva. Second, it promotes a restitutio ad integrum of the tissue, limiting the risk of blemishes, retracting scars, or even keloids. ${ }^{22}$ Third, it is favorable for patients because discomfort after intervention is reduced. Moreover, if stitches are not resorbable, a further follow-up is needed, which can be problematic with old patients or people with physical or physiological disabilities. ${ }^{23,24}$ Ultimately, avoiding sutures represents an economic advantage.
Considering the postoperative bleeding over time, all three techniques can be considered valuable devices and can be taken into consideration for patients suffering from bleeding diathesis or on medications affecting bleeding control. In fact, although biopsy is not mandatory with benign lesions of reactive etiology, for suspected premalignant or malignant affections, an investigative biopsy is compulsory. ${ }^{25}$

Undoubtedly, the postoperative pain score has to be considered one of the most important elements for the success of a biopsy technique. In this study, we have monitored postoperative pain through VAS and evaluating painkillers' assumption over time. ${ }^{26}$ Analyzing VAS values, the registration of pain over time revealed minimum symptoms for all three surgical techniques. This means that patients experienced minimum discomfort and improved life quality. Clinical studies have demonstrated that biopsy performed with laser and QMR are usually associated with reduced pain compared with the cold blade. Pogrel et al. hypothesized that the limited sensation of pain after laser surgery is due to the light's action on the vascular and lymphatic vessels. Laser light can seal vessels, limiting the discharge of fluids and molecules that are responsible for the starting of an inflammatory process. Consequently, edema and inflammation, together with pain, are reduced. ${ }^{27}$ This process stimulates the production of endorphins and determines and blocks nerve impulses carrying the sensation of pain. ${ }^{28,29}$

With respect to painkillers assumption, at $\mathrm{T} 2$ only 13 patients in the fully classified sample assumed them: to note, the lowest number was reported in the BLUE group, whereas the highest was 
in the QMR group. Paradoxically at T7, three patients belonging to the IR group still felt the necessity of taking painkillers. No patients took medications on T14 and T30. Despite the differences not being statistically significant, immediately after surgery (T2), the BLUE laser is considered the most painless technique, and this represents an excellent advantage for dealing with certain categories of patients such as pediatrics, patients with psychical disabilities, or phobic patients. ${ }^{30}$ One of the reasons why the BLUE laser is associated with minimum postoperative discomfort is related to its minimal thermal damage on tissues $(71.3 \pm 51.8 \mu \mathrm{m})$. Since histological alterations are limited, reduced local inflammation is obtained. Using the QMR scalpel, we generate a greater local trauma with consequently increased postoperatory edema.

Based on clinical evidence, laser devices are easier to use than QMR scalpels, which have a sharper learning curve rate. Moreover, the blue diode laser allows operators to completely avoid local bleeding, thus reducing the risk of anatomical structures damage (vessels and nerves) especially when operating in certain fields (floor of the mouth, palate, and ventral tongue). Before translating this device into the clinical practice, one of the main concerns was the risk of damaging the sample, thus impeding an accurate and reliable histopathological examination. In our cohort of patients, the BLUE group achieved the lowest level of thermal damage, and, in 15 cases, the pathologist was not able to report the entity of thermal damage as it was unperceivable. Although this aspect can be negligible when excising benign lesions, it can become relevant when a biopsy of malignant lesion is performed: being able to avoid the risk of thermal damage on the sample, it represents a great advance in the oral medicine and oncology fields.

This study confirms that this new technology accounts for the numerous benefits we have discussed and could largely be employed in the daily practice of surgeons. To further analyze these implications, it would be of great interest to evaluate other parameters such as duration of intervention, quality of life through a dedicated questionnaire, and patients' satisfaction. These topics will certainly be considered in future studies.

\section{Conclusion}

The results of our study support the concept that the introduction of this new technology in daily clinical practice could largely change and ameliorate the quality of surgical procedures. Its hemostatic effect is a major advantage, since it allows optimal visibility and reduces the necessity of sutures. These advantages lead to good compliance of the patient and excellent healing results. This device can be easily employed by nonexpert operators since the learning curve is lower compared with the QMR scalpel. Finally, the blue laser allows great advantages for the reduced risk of artefacts and damages of the sample for histopathological examination. This technique offers interesting fields of application in oral medicine and pathology and may become a suitable and safe instrument for private practitioners during their daily practice.

\section{Ethical Standard}

Human studies have been approved by the appropriate ethics committee and have therefore been performed in accordance with the ethical standards laid down in the 1964 Declaration of Helsinki and its later amendments. All persons gave their informed consent prior to their inclusion in the study.

\section{Disclosures}

G. Ottaviani has part-time employment in K-Laser d.o.o. (Sežana, Slovenia).

\section{Acknowledgments}

No funding was received to perform this study.

\section{References}

1. A. Pakfetrat et al., "Removal of refractory erosive-atrophic lichen planus by the $\mathrm{CO}_{2}$ laser," Oral Health Dent. Manage. 13(3), 595-599 (2014).

2. T. Bensaha, "A new approach for the surgical exposure of impacted canines by ultrasonic surgery through soft tissue," Int. J. Oral Maxillofac. Surg. 42(12), 1557-1561 (2013).

3. R. Broccoletti et al., "Quantic molecular resonance scalpel vs traditional scalpel in the treatment of labial mucocele: a two-center randomized controlled trial," Quintessence Int. 45(4), 331-338 (2014).

4. P. Vescovi et al. "Quantic molecular resonance scalpel and its potential applications in oral surgery" Br. J. Oral Maxillofac. Surg. 46(5), 355357 (2008).

5. C. Fornaini et al., "Four different diode lasers comparison on soft tissues surgery: a preliminary ex vivo study," Laser Therapy 25(2), 105-114 (2016).

6. D. De Santis et al., "Nd-YAP laser assisted frenulectomy: a case series on 23 patients," Minerva Stomatol. (2013).

7. A. Galanakis et al., "Focal epithelial hyperplasia in a human immunodeficiency virus patient treated with laser surgery," World J. Clin. Cases 2(7), 293-296 (2014).

8. G. E. Romanos, "Diode laser soft-tissue surgery: advancements aimed at consistent cutting, improved clinical outcomes," Compend. Contin. Educ. Dent. 34(10), 752-757 (2013).

9. N. Mirbehbahani and A. Rashidbaghan, "Treatment process for capillary hemangioma," Iran. J. Pediatr. Hematol. Oncol. 4(3), 127-130 (2014).

10. P. Vescovi et al., "Nd:YAG laser versus traditional scalpel. A preliminary histological analysis of specimens from the human oral mucosa," Lasers Med. Sci. 25(5), 685-691 (2010).

11. C. Fornaini et al., " $450 \mathrm{~nm}$ blue laser and oral surgery: preliminary ex vivo study," J. Contemp. Dent. Practice 17(10), 795-800 (2016).

12. C. Fornaini, J. P. Rocca, and E. Merigo, " $450 \mathrm{~nm}$ diode laser: a new help in oral surgery," World J. Clin. Cases 4(9), 253-257 (2016).

13. J. Reichelt et al., "A novel blue light laser system for surgical applications in dentistry: evaluation of specific laser-tissue interactions in monolayer cultures," Clin. Oral Invest. 21(4), 985-994 (2017).

14. V. G. A. Suter, S. Sjölund, and M. M. Bornstein, "Effect of laser on pain relief and wound healing of recurrent aphthous stomatitis: a systematic review," Lasers Med. Sci. 32(4), 953-963 (2017).

15. L. M. Aaltonen et al., "Voice quality after treatment of early vocal cord cancer: a randomized trial comparing laser surgery with radiation therapy," Int. J. Radiat. Oncol. Biol. Phys. 90(2), 255-260 (2014).

16. R. J. Turner et al., "Analysis of tissue margins of cone biopsy specimens obtained with 'cold knife,' $\mathrm{CO}_{2}$ and Nd:YAG lasers and a radiofrequency surgical unit," J. Reprod. Med. 37(7), 607-610 (1992).

17. S. J. Vore et al., "Comparative healing of surgical incisions created by a standard 'bovie,' the Utah medical epitome electrode, and a BardParker cold scalpel blade in a porcine model: a pilot study," Ann. Plast. Surg. 49(6), 635-645 (2002).

18. P. F. Bradley, "A review of the use of the neodymium YAG laser in oral and maxillofacial surgery," Br. J. Oral. Maxillofac. Surg. 35(1), 26-35 (1997).

19. R. S. Fernando and C. Muthu, "Adoption of endovenous laser treatment as the primary treatment modality for varicose veins: the Auckland City Hospital experience," N. Z. Med. J. 127(1399), 43-50 (2014).

20. M. Hochman, "Management of vascular tumors," Facial Plast. Surg. 28(6), 584-589 (2012).

21. A. Galanakis et al., "Focal epithelial hyperplasia in a human immunodeficiency virus patient treated with laser surgery," World J. Clin. Cases 2(7), 293-296 (2014).

22. T. M. Shin and J. S. Bordeaux, "How suture technique affects the cosmetic outcome of cutaneous repairs," J. Drugs Dermatol. 13(8), 967969 (2014). 
23. P. Vescovi et al., "L'impiego del bisturi a risonanza quantica molecolare nei settori estetici del cavo orale," Quintessenza Int. 2, 39-45 (2005).

24. M. C. M. Haytac and O. O. Ozcelik, "Evaluation of patient perceptions after frenectomy operations: a comparison of carbon dioxide laser and scalpel techniques," J. Periodontol. 77(11), 1815-1819 (2006).

25. M. Agarwal et al., "Management of the dental patient on anticoagulant medication: a review," N. Y. State Dent. J. 80(4), 29-32 (2014).

26. K. Isik et al., "Comparison of three pain scales after impacted third molar surgery," Oral Surg. Oral Med. Oral Pathol. Oral Radiol. Endodontol. 112(6), 715-718 (2011).

27. M. A. Pogrel, C. K. Yen, and L. S. Hansen, "A comparison of carbon dioxide laser, liquid nitrogen cryosurgery, and scalpel wounds in healing," Oral Surg. Oral Med. Oral Pathol. 69(3), 269-273 (1990).

28. S. A. Kahraman, "Low-level laser therapy in oral and maxillofacial surgery," Oral Maxillofac. Surg. Clin. North Am. 16(2), 277-288 (2004).

29. D. M. Ferreira et al., "Analgesic effect of He-Ne $(632.8 \mathrm{~nm})$ low- level laser therapy on acute infammatory pain," Photomed. Laser Surg. 23(2), 177-181 (2005).

30. L. A. Kotlow, "Oral diagnosis of abnormal frenum attachments in neonates and infants: evaluation and treatment of the maxillary and lingual frenum using the erbium: YAG Laser," J. Pediatr. Dent. Care 10(3), 1114 (2004).

Margherita Gobbo graduated in dentistry with full marks and honors in 2011. She received her master of science with full marks and honors in 2014. She completed a scholarship in 2015 regarding the use of laser therapy in side effects of oncological therapies. She collaborates with the Division of Oral Medicine and Pathology (Trieste) dedicating mainly to oncological and special needs patients. She has participated in numerous national and international conferences and authored several publications.

Rossana Bussani graduated in medicine in 1984. She received her master of science in pathology in 2008. She is an associate professor at the University of Trieste since 2002. She is involved in several research projects and is entitled to the teaching of pathology in diverse degree courses including medicine, dentistry, and dental hygiene. She has participated in numerous national and international conferences and has authored several publications.

Giuseppe Perinetti graduated in dentistry in 2000. He received his $\mathrm{PhD}$ in cell biology in 2009 and a master of science in orthodontics in 2011. He collaborates with the University of Trieste since 2009 serving as a consultant for the statistical analysis of numerous papers. He has participated in several national and international conferences and has authored several publications.

Katia Rupel graduated in biology in 2008 and in dentistry with full marks and honors in 2013. She collaborates with the Division of Oral Medicine and Pathology (Trieste) dedicating mainly to oncological and special needs patients. She is completing a $\mathrm{PhD}$ in nanotechnology. She has participated in numerous national and international conferences and has authored several publications.

Lorenzo Bevilacqua graduated in dentistry in 2000 . He received his master of science in oral surgery. He is employed as a researcher at the University of Trieste and coordinated the student of the degree course in oral hygiene. He has participated in numerous national and international conferences and has authored several publications.

Giulia Ottaviani graduated in dentistry with full marks and honors in 2011. She received her $\mathrm{PhD}$ in nanotechnology in 2015 and a European master of science in laser in dentistry in 2016. She is currently enrolled in a master of science in oral surgery and collaborates with the Division of Oral Medicine and Pathology (Trieste) dedicating mainly to oncological and special needs patients. She has participated in numerous national and international conferences and has authored several publications.

Matteo Biasotto graduated in dentistry in 1997. He received his $\mathrm{PhD}$ in dental materials in 2002 and a master of science in oral surgery in 2007. He is an associate professor at the University of Trieste. He is the chief of the Division of Oral Medicine and Pathology of Trieste. He is director of the degree course of dentistry in Trieste. He has participated in numerous national and international conferences and authored several publications. 